\title{
Tangence
}

\section{La littérature régionaliste en France (1900-1940)}

\section{Anne-Marie Thiesse}

Numéro 40, mai 1993

Régionalismes littéraires de la francophonie

URI : https://id.erudit.org/iderudit/025766ar

DOI : https://doi.org/10.7202/025766ar

Aller au sommaire du numéro

Éditeur(s)

Tangence

ISSN

0226-9554 (imprimé)

1710-0305 (numérique)

Découvrir la revue

Citer cet article

Thiesse, A.-M. (1993). La littérature régionaliste en France (1900-1940).

Tangence, (40), 49-64. https://doi.org/10.7202/025766ar d'utilisation que vous pouvez consulter en ligne.

https://apropos.erudit.org/fr/usagers/politique-dutilisation/ 


\section{La littérature régionaliste en France (1900-1940) Anne-Marie Thiesse *}

La France est le pays par excellence du centralisme. Cela vaut pour la vie politique, économique, administrative et peut-être plus encore pour le domaine culturel. La concentration de la vie littéraire et artistique dans la Capitale est extrême: là se trouvent les salons, les cafés, les journaux, les institutions qui assurent la reconnaissance et font les classements esthétiques. Cette hégémonie parisienne, que le processus d'autonomisation du champ littéraire n'a fait que renforcer, peut-elle être battue en brèche? Tel est précisément le but affiché par les inventeurs et les promoteurs du régionalisme, un mouvement peu connu mais important de la première moitié du $x^{e}$ siècle.

Ce terme de régionalisme apparaît dans la langue française seulement à la fin du $x x^{e}$ siècle. Mais il connaît très vite un large succès et on le retrouve en abondance sous la plume des critiques et essayistes littéraires de la Troisième République. Les *Panoramas de la littérature française contemporaine "publiés dans l'entredeux-guerres présentent généralement une rubrique "littérature régionaliste. Mais il serait assez vain de chercher, au moyen d'éventuels critères internes, l'essence du régionalisme comme genre littéraire (même les créateurs du terme régionalisme ont toujours été fort imprécis sur sa définition). Il s'agit en fait d'une tentative, d'assez longue durée, visant à constituer un "contrechamp" littéraire et à introduire d'autres critères de légitimité 1 .

\section{La Province contre Paris}

La fin du XIX ${ }^{e}$ siècle voit naître ce qui, vu de Paris, s'appelle le "Réveil des Provinces". La Province française s'est enrichie, le

- Anne-Marie Thiesse est chercheure au CNRS à Paris. Elle a publié Le roman au quotidien. Lecteurs et lectures populaires à la Belle Epoque (1984) et Ecrire la France. Le mouvement littéraire régionaliste de langue francaise entre la Belle Epoque et la Libération (1991).

1 Pour une présentation plus générale du régionalisme, voir Anne-Marie Thiesse, Ecrire la France, le mouvement littéraire régionaliste en France de la Belle Epoque à la Libération Paris, Presses universitaires de France, 1991. 
50

parlementarisme lui donne une animation politique, la presse locale se développe et se diversifie. La relative croissance des universités et des établissements d'enseignement secondaire permet aux jeunes "élites locales" de former des groupes d'amis partageant goûts et ambitions. C'est l'époque où se multiplient les écoles" esthétiques qui essayent de se faire connaitre par la publication de manifestes et le lancement de revues. Phénomène nouveau et notable: le mouvement ne touche pas seulement les rives de la Seine, mais aussi les villes de province. En une dizaine d'années, des revues littéraires "de jeunes * éclosent un peu partout en France, au premier chef dans les grandes villes universitaires (Toulouse, Marseille, Lille, etc.) mais aussi dans des cités plus modestes. Les revues publient les essais poétiques de leurs créateurs (elles sont souvent doublées de maisons d'édition pratiquant le compte d'auteur), des articles sur la littérature de l'avenir et l'état présent de la Nation, des critiques littéraires, etc. Très vite naissent entre ces revues des relations transrégionales (échanges d'articles, publicités mutuelles) et on y voit s'exprimer de plus en plus explicitement la volonté de fédérer les jeunes énergies provinciales pour lutter contre le centralisme.

Plusieurs "enquêtes sur la décentralisation littéraire " sont également lancées, souvent à l'initiative de directeurs de quotidiens provinciaux. Les réponses, en général, proposent des moyens pour lutter contre l'hégémonie parisienne en s'appuyant sur une vaste fédération transrégionale des provinciaux:

Il existe un trust formel ou tacite des puissances de la capitale, alliées souvent à la puissance des capitaux, contre toute ouvre ne portant pas l'estampille du Syndicat organisé entre auteurs, critiques et éditeurs d'une part, théâtres, music-halls, salons, etc. Ensemble formant une Église infaillible hors de laquelle il n'est point de salut, un pontificat décrétant l'excommunication majeure, ou l'ilotisme de la Province. [...] Il faut emprunter la force même du vainqueur en faisant de Paris le centre commercial des œuvres de décentralisation. [...] Il faut prendre Paris pour ce qu'il est réellement: le marché littéraire et artistique, comme il est celui du beurre, des oufs et de la marée. [...] Les groupements provinciaux doivent se constituer en une fédération effective et puissante, avec l'appui des provinciaux arrives à Paris. [...] À la puissance d'un Syndicat, on ne peut opposer que celle d'un autre Syndicat. ${ }^{2}$

2 Achille Magnier, dans J.R. Aubert, Enquête sur la décentralisation littéraire, Paris, Bibliothèque de l'Association, 1904. 
Se grouper en Fédération avec l'appui des provinciaux arrivés à Paris... C'est en fait à peu près ce qui se passe alors. De jeunes provinciaux (ils ont entre 20 et 30 ans) installés récemment à Paris avec l'ambition de faire une carrière nationale, politique ou culturelle, agitent depuis le milieu des années 1890 l'idée d'une Fédération des jeunes énergies provinciales pour lutter contre le centralisme et l'hégémonie parisienne. Dans la mesure où ils entendent se situer au delà des partitions politiques et idéologiques existantes, ils donnent à leur mouvement l'appellation de - régionalisme *: le terme étant tout à fait neuf, et donc dépourvu de connotation, on peut aisément en faire un terme consensuel. Le nouveau vocable est très vite lancé:

Le régionalisme est à la mode. Inconnu hier encore, le mot a fait une fortune rapide. On ne compte plus les groupements, les revues, les journaux, les théâtres, les romans régionalistes. [...] Néanmoins, parmi cette vogue, la notion du régionalisme reste embrouillée, et le terme recouvre les plus diverses conceptions. $^{3}$

L'auteur de ce constat est, précisément, le grand propagateur et théoricien du régionalisme, Jean Charles-Brun. Né à Montpellier en 1870, professeur agrégé de lettres et membre du Félibrige ${ }^{4}$, il est la cheville ouvrière du groupement politique créé en 1900 sous le nom de Fédération régionaliste française, qui a pour vocation de mener la lutte contre le centralisme français. Charles-Brun est aussi l'auteur d'innombrables articles sur le régionalisme et d'un gros ouvrage qui veut être la présentation exhaustive des idées régionalistes en matière politique, économique et culturelle (Le régionalisme, Bloud et cie, 1911). Il l'a fait précéder d'un autre volume intitulé Les littératures provinciales (Bloud et cie, 1908). On voit par là que les thèmes régionalistes sont développés simultanément dans les domaines politiques et littéraires. Développés plutôt que définis: en effet, si le mal (le centralisme) est clairement désigné et ses méfaits longuement

Charles-Brun, Le régionalisme, [s.l.], Bloud et cie, 1911, $2^{\epsilon}$ éd., p. 1-5.

4 Créé au milieu du XIX' siècle, le Félibrige est un mouvement de défense et d'illustration de la langue d'oc. Les félibres veulent développer une littérature de qualité en langue d'oc, épurer l'idiome populaire et développer la connaissance des traditions rurales dans le Midi de la France (création d'un Armana Prouvençau, du Museon Arlaten par Frédéric Mistral). À la fin du $\mathrm{XIX}^{\mathrm{e}}$ siècle, le Félibrige rassemble nombre de membres de la petite et moyenne bourgeoisie dans les régions du Sud de la France. 
52

exposés, les remèdes proposés correspondent plutôt à un vaste catalogue de réformes souhaitées, parfois contradictoires, sans grand souci d'entrer dans les détails pratiques qui pourraient être sources de clivages idéologiques. En fait, l'argumentaire régionaliste correspond approximativement au raisonnement suivant: la France, en ce tournant du siècle, est grandement menacée par la décadence; la maladie pernicieuse qui la mine, c'est le centralisme, auquel on peut imputer à peu près tous les problèmes présents et à venir; ergo, en luttant contre le centralisme, c'est-à-dire en œuvrant pour le régionalisme, on accomplira le devoir patriotique suprême. Dans le domaine littéraire, la démarche est similaire. La littérature française, disent les régionalistes, est menacée de ruine par le déferlement d'une production parisienne déliquescente et amorale; heureusement les sources vives de l'esprit français existent toujours, dans la fraîcheur intacte des provinces; y régénérer l'esprit français est l'œuvre de salut public qui s'impose. Soit, comme le dit un contemporain:

Si le roman régional a la prétention de réagir contre le roman parisien, qu'il soit loué, cent fois loué! Qui donc en effet nous délivrera de toute cette littérature où l'adultère, le crime, le cambriolage et le reste jouent un si grand rôle aujourd'hui? Estce que l'esprit français serait incapable de trouver autre chose? Et l'esprit provincial n'est-il pas assez sain, assez vigoureux pour renouveler et purifier l'art d'écrire des romans? 5

La littérature régionaliste doit donc être le souffle d'air frais qui purifie la littérature française en balayant les miasmes des alcôves. Les métaphores hygiénistes (hygiène sociale comprise) abondent sous la plume des militants régionalistes. Paris, prototype de la mégalopole, est dans ces déclarations le lieu de toutes les contagions et de toutes les dégradations.

La pléthore de médiocrités qui encombrent les temples de l'art et de la littérature n'a d'autre cause que la centralisation outrancière du $\mathrm{XIX}^{\mathbf{e}}$ siècle. Sur le cerveau des hommes du terroir affamés d'idéal, Paris agissait à distance comme la lumière sur un papillon; et hélas, ils sont légions ceux qui s'y sont brûlé les ailes; pour un succès, que de déboires ... ${ }^{6}$

5 Jean Amade, L'idèe régionaliste, Perpignan, Éditions de la Bibliothèque Catalane, 1912, p. 151.

6 Paul Gourmand, dans J.R. Aubert, Enquête sur la décentralisation littêraire, op. cit., 1904. 
La Province, les campagnes, à l'inverse, seraient les lieux de cure par excellence. Depuis que l'industrialisation, vidant les campagnes de leur trop-plein de misérables et révoltés potentiels, a déporté le péril social des villages sur les villes, les paysans sont devenus, dans l'imaginaire social, le Peuple parfait. La vertu éternelle des champs, donc, contre la modernité urbaine et le mouvement ouvrier? De ces déclarations enflammées contre les plaisirs urbains, certains critiques ont conclu, trop rapidement, que le régionalisme n'était qu'une des idéologies de la droite, conservatrice ou réactionnaire, appuyant l'ordre établi, le sabre et le goupillon. Certes, les idéologues de droite ne sont pas dénués d'intérêt pour le régionalisme et quelques-uns s'en font les champions. Mais le régionalisme ne se confond pas avec une célébration nostalgique de la France rurale, vertueuse et soumise, éventuellement monarchiste. Une autre démarche est aussi à l'œuvre, dans le mouvement régionaliste, qui critique les hiérarchies en place et la légitimité culturelle.

\section{Retourner le handicap}

Les écrivains régionalistes sont des provinciaux qui ont voulu faire carrière dans les lettres en adoptant les genres et les styles alors les plus prestigieux pour les débutants: la plupart, au tournant du siècle, s'essaient au lyrisme post-symboliste. Mais la concurrence est sévère et ces provinciaux ne sont pas les mieux placés pour comprendre les règles et les usages des cénacles et des salons littéraires. Bien souvent, leur ignorance les place dans des situations embarrassantes et humiliantes où ils se voient taxés de paysans balourds. Les railleries à propos de leur accent régional, de leurs manières "rustiques" ne leur sont pas épargnées. L'adoption d'une revendication régionaliste consiste en un retournement de ce stigmate initial. Certes, disent-ils, nous faisons "Peuple, nous passons pour des paysans: c'est que nous représentons précisément, ce Peuple paysan dont la culture, en fait, est infiniment plus respectable que les raffinements décadents et cosmopolites de la Capitale.

Ainsi en est-il pour Louis Pergaud, qui ne songeait pas, initialement, qu'il pût connaître le succès par des romans villageois. Fils d'instituteur rural, et brillant élève de l'École Normale d'instituteurs de Besançon, Louis Pergaud avait commencé très jeune à publier des poèmes fort maniérés à compte d'auteur. Mais il clevait gagner 
sa vie comme instituteur dans un village de montagne: le jeune instituteur socialiste n'avait pas alors de mots assez durs pour exprimer sa haine de la "populace arriérée * et bigote au milieu de laquelle il se trouvait. Réussissant finalement à s'échapper vers la Capitale, où il rêve de participer à la vie littéraire, il ne tarde pas à déchanter. Dans ses conversations avec les écrivains qu'il arrive à fréquenter, il se sent gauche, terne. Son allure, sa tenue font rire:

Venu de sa Franche-Comté natale à Paris, où il était instituteur et où il prenait sur ses veillées pour avancer ses travaux littéraires, Louis Pergaud demeurait «paysan»; il habitait notre quartier, du côté de l'église de Montrouge et se trouvait dépaysé dans ce coin de la capitale, pourtant encore rustique. [...] Il ne savait où placer son grand corps gauche et ses énormes pieds chaussés de prodigieuses "godasses". Il se dégageait cependant de lui une mâle odeur de terroir, une manière de franchise abrupte et directe. [...] il était peu au courant des mystères et des coulisses de la comédie littéraire et faisait parfois sourire par des naïvetés qui illustraient seulement la candeur de cet esprit pur, de ce cœur simple. ${ }^{7}$

Progressivement, l'œuvre de Pergaud se met à coïncider avec l'image qu'ont de lui les littérateurs parisiens. Délaissant le lyrisme pour la prose, il rédige des récits animaliers puis des romans portant sur l'enfance rurale et il adopte, pour présenter son œuvre, l'argumentaire régionaliste :

J'étais à peu près sûr que La guerre des boutons vous plairait. J'en avais, au préalable, essayé quelques chapitres sur quelques amis intelligents, sains, dont le goût bien français m'inspire toute confiance. L'épreuve avait été satisfaisante.

Que cela ne plaise pas aux cervelles légères des boulevardiers qui préfèrent les idylles se déroulant sur les canapés des maisons hospitalières, c'est fort possible, c'est même probable, mais [...] j'espère qu'il se trouvera encore un public français si restreint soit-il — pour goûter le charme de cette épopée. ${ }^{8}$

Lauréat du prix Goncourt en 1910 pour De Goupil à Margot, Louis Pergaud connaît un large succès pour ses romans. Son décès en 1915, sur le front, interrompt précocement sa carrière.

7 Pierre Descaves, Mes Goncourts, Paris, Laffont, 1944, p. 154. Pierre Descaves est le fils de Lucien Descaves, l'un des premiers membres de l'Académie Goncourt, qui soutiendra activement Pergaud dans ses débuts romanesques.

8 Lettre de Louis Pergaud à Edmond Rocher, 18 août 1912. 
La contestation de la hiérarchie esthétique, on le voit bien à cet exemple, passe par l'introduction, dans le champ littéraire, de critères hétéronomes, éthiques. Confrontés à un système de classement qui les place dans une situation d'infériorité, ou les exclut (c'est encore plus le cas pour les écrivains-paysans), les individus éloignés par leurs origines des milieux littéraires ne peuvent que se référer à des valeurs "impures " dans un champ où la légitimité "purement" esthétique est fortement mais implicitement corrélée à la hiérarchisation sociale. Cependant, à la différence de l'argumentaire développé ultérieurement par les écrivains prolétariens, le discours régionaliste ne s'en prend pas directement à la hiérarchie sociale; plus exactement, il la traite par métonymie. Paris étant assimilé au pouvoir, au suprême degré de la hiérarchie en tout domaine, et la Province, a contrario, représentant les dominés, la lutte entre le haut et le bas social est en fait traitée comme une lutte entre la Capitale et le reste de la France. On s'en doute, cette manière agéographique * de présenter le conflit a plus de chance de rencontrer un certain consensus qu'une mise à jour directe des conflits de classe dans le champ culturel. L'ambiguîté du régionalisme assure son efficacité; certes, il n'y aura pas de changement révolutionnaire dans le champ littéraire mais la critique accueillera volontiers cette production littéraire en reprenant les étiquettes flatteuses qu'elle s'attribue.

\section{La percée régionaliste}

La réussite du régionalisme tient aussi à ce qu'il joue de la stratégie de groupe. Au lendemain de la Première Guerre mondiale, une Société des Écrivains de Province est fondée, avec vocation d'organiser matériellement et intellectuellement la solidarité entre écrivains provinciaux. La Société publie une revue, la Renaissance provinciale, qui fonctionne un peu comme un bulletin *d'admiration mutuelle . Régulièrement sont présentées des biographies et des critiques élogieuses des membres de l'Association. Les extraits d'œuvre et les essais régionalistes sont nombreux: ils illustrent plus particulièrement les mœurs, l'âme ou les paysages d'une région. En 1924, la Renaissance Provinciale lance le projet de constituer une "Académie Goncourt de Province . Le démarquage voulu de l'Académie Goncourt est significatif. Pour faire pièce à l'Académie française qui donnait pour illégitime le roman, et plus encore le réalisme, Jules et Edmond de Goncourt avaient créé une contre-Académie, composée 
56

de romanciers réalistes. La Société des Écrivains de Province reproduit très exactement cette stratégie offensive des exclus élaborant eux-mêmes l'instance susceptible de les reconnaître.

La composition de l'Académie de Province résulte d'un vote organisé parmi les écrivains provinciaux, invités à désigner dix d'entre eux, âgés de plus de cinquante ans. La première Académie se compose de: François Fabié (Rouergue), CharlesThéophile Féret (Normandie), Émile Guillaumin (Bourbonnais), Philéas Lebesgue (Picardie), Anatole Le Braz (Bretagne), Gabriel Maurière (Champagne), Henry Mériot (Saintonge), Achille Millien (Nivernais), Joseph de Pesquidoux (Armagnac) et Gabriel Sarrazin (Saintonge). À peine formée, l'Académie se voit investie de la mission de décerner un prix littéraire annuel. Il ne s'agit pas de couronner un ouvrage publié mais de désigner un manuscrit que les Éditions du Fleuve à Lyon se proposent d'éditer. L'accent est donc délibérément mis sur le soutien aux débutants condamnés à la publication *à compte d'auteur .

Parallèlement à cette entraide mutuelle organisée, fonctionne aussi entre écrivains régionalistes tout un réseau informel de soutien: les plus reconnus parrainent leurs cadets auprès de maisons d'édition, leur consacrent des critiques dans la presse provinciale ou nationale, leur adressent conseils et encouragements. Le régionalisme littéraire ne constitue pas une école littéraire (il n'a pas de chef, ni de programme esthétique précis), mais bien plutôt une nébuleuse assez vaste où prédominent les relations directes.

La reconversion du jeune Pergaud, passant de la poésie éthérée au roman rustique et provincial, est en effet loin de constituer un cas unique. Entre le début du siècle et la Seconde Guerre mondiale, les romans qualifiés de régionalistes se comptent par centaines, si ce n'est par milliers. C'est dire qu'ils rencontrent la faveur du public. En fait, les publications régionalistes constituent les gros bataillons de ce que l'on pourrait appeler le secteur moyen de la production littéraire, entre l'avant-garde esthétique et la littérature populaire. Les éditeurs nationaux prennent acte de cet engouement en créant des collections régionalistes; il y aura même dans les années 1920 à la NRF une collection de "Livres du Pays": il est vrai que la rubrique "Paris " y est plus abondante que les rubriques "Périgord " ou "Quercy"! Les prix littéraires qui sont créés durant cette période couronnent souvent des œuvres régionalistes. Le Goncourt va en 1907 à Terres lorraines (Émile Moselly), en 1911 à Monsieur des Lourdines 
(Alphonse de Chateaubriant), en 1912 à Filles de la pluie (André Savignon), en 1913 au Peuple de la mer (Marc Elder), en 1920 à Nêne (Ernest Pérochon), etc. Le Fémina est décerné en 1918 au Serviteur (Henri Bachelin), en 1921 à Cantegril (Raymond Escholier), en 1927 à Grand-Louis l'Innocent (Marie Le Franc) et plusieurs prix Renaudot des années 1920 sont également régionalistes.

\section{La représentation du monde rural et provincial}

Il semble que le régionalisme s'inscrive dans le sillage du réalisme et du naturalisme, reprenant ce qui a fait le succès de ces courants, à savoir l'exploration, ici dans les seules provinces, du monde social. Les intrigues de ces romans sont souvent simples: à la différence des romans populaires, il n'y a guère ici de péripéties haletantes. Les ressorts narratifs sont élémentaires: l'amour, la haine, les coups du sort. L'intrigue est parfois si ténue qu'elle apparaît comme le simple fil conducteur de descriptions et de notations. Plus ou moins clairement, la plupart des romans ont pour véritable ressort dramatique l'opposition entre ville et campagne. Certains la traitent de manière manichéenne: séduits un temps par les mirages de la ville, les héros positifs finissent par y renoncer et par confirmer leur appartenance définitive au village et à la terre. Les romanciers catholiques, comme Charles Silvestre, se spécialisent dans la dénonciation de l'exode rural et les proclamations de fidélité à la vie rurale, parée de toutes les vertus. Mais souvent les romanciers font une peinture beaucoup plus noire de la vie rurale et mettent en lumière les différences de condition sociale ou de religion qui divisent des communautés que les observateurs bien-pensants s'obstinent à dire harmonieuses. Les instituteurs auteurs de romans régionalistes (Ernest Pérochon, Nêne, Goncourt 1920, Jean Rogissart, Mervale, Renaudot 1937, ou encore Lucien Gachon, Maria, Jean-Marie), donnent généralement une vision assez noire des haines et des jalousies qui traversent les sociétés rurales. Mais le monde villageois apparaît toujours, in fine, comme moins redoutable que la ville corruptrice et engloutissante, où l'on se perd irrémédiablement.

La description souvent précise des usages ruraux et provinciaux donne un intérêt tout particulier à ces romans. Les notations de type ethnographique sont nombreuses et la prose régionaliste fournit un riche florilège des grands moments de la 
vie rurale: mise à mort du cochon, charivaris, fêtes traditionnelles, etc. Un certain nombre d'auteurs, d'ailleurs, publient des travaux folkloristes en sus de leur œuvre romanesque. En fait, plus que les publications ethnographiques proprement dites dont la diffusion était restreinte, les romans régionalistes ont fait connaître à un large public les sujets de prédilection des folkloristes.

Le style de ces récits est en bonne partie inspiré des romans du $\mathrm{XIX}^{\mathrm{e}}$ siècle et le démarquage de l'êcriture réaliste ou naturaliste est souvent évident.

L'air était vif et jeune; la terre fumait. Derrière le versoir mille petites haleines fusaient, droites, précises, subtiles; elles semblaient vouloir monter très haut comme si elles eussent été heureuses d'échapper enfin au poids des mottes et puis elles se rabattaient et finissaient par s'étendre en panaches dormants. Le souffle oblique des boufs précédait l'attelage et remontait, couvrant les six bêtes d'une buée plus blanche qu'agitaient des tourbillons de mouches. ${ }^{9}$

Dans la plupart des cas, les auteurs tentent de donner l'impression, par leur écriture, du parler populaire régional. Ces tentatives s'exercent à divers degrés. Parfois l'écrivain se contente d'introduire un peu de "couleur locale " par l'utilisation de termes techniques ou régionaux, éventuellement explicités par des notes en bas de page. Toléré lorsqu'il est modéré, l'usage de termes régionaux déchaîne la critique quand l'êcrivain y recourt abondamment. Alphonse de Chateaubriant, notamment, reçut de nombreux reproches pour avoir cité dans La Brière un grand nombre de mots du cru. Il dut présenter un argumentaire de défense:

Je n'ai pas choisi ma langue, elle m'a été imposée par la vie profonde du sujet qui m'a dominé de son exactitude. Ces termes techniques locaux, qui sont d'ailleurs en petit nombre, je n'avais ni le droit ni la possibilité de les rejeter: je n'avais pas d'équivalent en français. Priver mon texte de ces mots si chargés de réalités terriennes, c'eût été les appauvrir. J'ai employé le mot salet, par exemple, qui, dans tous les ouvrages traitant de l'industrie de la motte, désigne l'outil qui sert à couper. Le mot marre, qui désigne également un outil tranchant de même usage, est un vieux mot français que vous trouvez dans Rabelais et qui n'a jamais cessé de figurer dans la langue du travail. ${ }^{10}$

9 Extrait de Nêne, d'Ernest Pérochon, Goncourt 1920.

10 Alphonse de Chateaubriant, dans Frédéric Lefèvre, Une beure avec... Alphonse de Chateaubriant, Paris, NRF-Gallimard, 1924, 1* série. 
Les écrivains justifient les recours à la langue populaire par une triple argumentation. Il n'y a pas, disent-ils, d'autres vocables pour désigner les objets et les usages présentés; en outre, ces mots ont toute la saveur, la couleur, l'âme du paysage régional; de surcroît, ces expressions que la langue relevée ignore appartiennent au français le plus originel, le plus authentique. D'une manière plus élaborée, quelques écrivains insèrent dans les dialogues des locutions, des dictons, des idiomatismes, voire une syntaxe populaire régionale. Mais l'insertion dans le récit à la troisième personne de la langue populaire, de son rythme et de son expressivité suppose une technique stylistique plus approfondie. L'usage du discours indirect libre, que Zola a grandement contribué à mettre au point dans la narration romanesque, autorise des tentatives plus ou moins audacieuses. Occasionnel dans la plupart des romans régionalistes, ce mode d'expression est beaucoup utilisé par Giono, et plus encore par Lucien Gachon.

\section{Le régionalisme comme identité française officielle}

Aussi paradoxal que cela puisse paraître, le régionalisme constitue dans la première moitié du $\mathrm{xx}^{\mathrm{e}}$ siècle une des représentations de l'identité nationale. Il est devenu manifeste, après la guerre perdue contre l'Allemagne en 1870, que la France n'était plus la plus puissante des nations, celle qui pouvait proposer (imposer) au monde ses valeurs et s'ériger en modèle universel. Une autre définition de la "francéité " s'est alors constituée, plus modeste, assurément, mais apparemment moins fragile, puisqu'elle entendait se fonder sur la France des terroirs ou plus exactement, des "petites patries", selon l'expression alors en usage. La grandeur de la France, dans le discours qui se met alors en place, ne réside pas dans une suprématie en tous domaines: elle tiendrait à ce que la France, dans sa diversité, réunit harmonieusement tout ce qui est nécessaire au bonheur humain. La France serait le résumé idyllique de l'Europe, une mosaïque parfaite des particularités complémentaires. Cette image de la France est déclinée par les manuels de géographie, de lecture, d'histoire pendant des décennies, selon une démarche dont le paradigme est offert par le célèbre Tour de France par deux enfants, rédigé après la guerre de 1870 par G. Bruno, épouse d'un idéologue républicain. Ce livre scolaire, lu par des millions d'enfants pendant plusieurs générations, apprend aux écoliers à chérir la Grande Patrie par la découverte émerveillée des petites 
60

patries. Et les manuels de lecture de l'école primaire, d'une manière générale, proposent aux enfants la découverte de la littérature française à travers des extraits d'œuvres régionalistes ${ }^{11}$.

Le régionalisme reçoit une consécration encore plus officielle dans les années 1930, en une période de crise économique et sociale extrême. La représentation de la nation comme union harmonieuse de terroirs, dans lesquels s'enracinerait le Peuple de France, permet d'exprimer un consensus minimal. C'est ainsi que la partie française de l'Exposition Internationale des Arts et des Techniques, qui se tient à Paris pendant l'été 1937, est placée sous le signe du régionalisme. Cette Exposition est la dernière grande manifestation internationale avant le déclenchement de la Seconde Guerre mondiale et constitue pour chaque pays représenté l'occasion d'affirmer son identité (les visiteurs se rappelleront tous la confrontation symbolique des pavillons imposants de l'Allemagne nazie et de l'Union soviétique). Le gouvernement français, alors de droite, avait choisi en 1935 cette invocation au régionalisme et la référence fut reprise par le gouvernement de Front Populaire, regroupant les mouvements de gauche, élu en 1936. Comme le déclarait le commissaire de l'Exposition, le régionalisme devait manifester l'union paisible de tous les Français:

L'Exposition de 1937 présentera aux yeux des visiteurs la France des métiers et des arts, la France des fêtes et des jeux, la France des chansons et des poèmes, la France des paysages. Et ainsi, tout en servant la cause de la province, elle symbolisera l'union de tous les Français * avec tous les traits d'une individualité régionale * qui nous est chère, suivant le mot de Georges Clemenceau. ${ }^{12}$

À l'occasion de l'Exposition, des bibliographies littéraires régionales sont éditées dans des brochures largement diffusées auprès des visiteurs. Il y a même, dans le cadre d'une exposition présentant un Musée de la Littérature française alors en projet ${ }^{13}$, une vaste présentation sur panneaux de l'histoire de la littérature française complètement réécrite dans une perspective régionaliste!

11 L'auteur de cet article, qui fut écolière dans les années 1960, apprit les subtilités orthographiques de la langue française à travers les œuvres de Pérochon, Pesquidoux, Pergaud et autres régionalistes.

12 Edmond Labbé, Le régionalisme et l'Exposition internationale de Paris 1937, Paris, Imprimerie nationale, 1936.

13 La réalisation de ce Musée de la Littérature sera ajournée par la Seconde Guerre mondiale, puis abandonnée. 
Trois ans plus tard, la défaite française et l'Occupation amenèrent au pouvoir, dans une France divisée en plusieurs zones un gouvernement réactionnaire. L'État français ayant pour chef le maréchal Pétain reprit d'emblée, comme mot d'ordre de sa *Révolution nationale, ce terme de régionalisme qui avait semblé si opportunément consensuel sous le régime républicain précédent. L'expression devint la bannière d'un régime pratiquant la collaboration active avec l'Allemagne nazie et le centralisme le moins démocratique. Le ruralisme le plus réactionnaire (avec le mot d'ordre du "Retour à la Terre ") fut l'idéologie officielle de ce régime. Les fêtes folkloriques en costumes, la célébration de l'artisanat traditionnel et du folklore furent le décor chatoyant d'une sinistre réalité où se multipliaient rafles de résistants et déportations de Juifs. Cependant rares furent les ouvres littéraires à caractère régionaliste publiées en France durant les années d'Occupation: le contingentement très strict du papier alloué à l'édition limitait fortement les publications et les autorités d'Occupation préféraient accorder leurs faveurs aux écrivains acceptant de collaborer plus ou moins activement avec l'Allemagne nazie (ils furent rares parmi les écrivains régionalistes). Seule initiative notable - et durable - du gouvernement pétainiste: la création d'un prix littéraire, dit prix Sully-Olivier de Serres ${ }^{14}$, destiné à "encourager, soutenir et récompenser la littérature consacrée à la vie paysanne.. Dans le jury figuraient des écrivains régionalistes (Charles-Brun, Lucien Gachon, Hugues Lapaire, Henri Pourrat, Charles Silvestre, Jean Yole, Philéas Lebesgue, etc.) aux côtés de Colette et du directeur du Musée des Arts et Traditions Populaires.

\section{Le particularisme et l'universalisme}

Après la Libération, le régionalisme eut à pâtir de cette appropriation du régionalisme par le gouvernement pétainiste. Le terme entre en grand discrédit et ne ressurgit sur la scène

14 Sully avait été ministre du roi Henri IV et tous les manuels d'histoire de la Troisième République lui avaient attribué la formule: "Labourage et paturage sont les deux mamelles de la France.. Son contemporain Olivier de Serres faisait figure dans ces mêmes manuels de premier agronome éclairé de la France. Le prix Sully-Ollivier de Serres est encore aujourd'hui décerné sous l'égide du ministère de l'Agriculture. Parmi les lauréats récents figure le Breton Pierre-Jakez Hélias dont le Cbeval d'orgueil fut un best-seller des années 1970. 
publique que dans les années 1970 , dans le sillage des mouvements de contestation du pouvoir qui se mettent en place après les événements de Mai 1968. Le régionalisme prend alors un tour idéologique d'extrême-gauche et adopte souvent une thématique tiers-mondiste (thème de la colonisation intérieure des régions françaises opprimées par l'impérialisme français); il se colore progressivement d'une tournure "rétro (redécouverte de l'agriculture traditionnelle éliminée par la modernisation, des vieilles coutumes, musiques et costumes) et préfigure certains aspects de l'écologisme actuel.

Cependant, dans le domaine littéraire, le régionalisme a perdu les positions conquises dans la première moitié du siècle. Ce n'est pas que les romans présentant la vie rurale ou provinciale, présente ou passée, aient aujourd'hui disparu: ils sont même encore nombreux et certains (que l'on songe aux œuvres de Bernard Clavel) ont de beaux succès en librairie. Mais l'étiquette régionaliste reste fortement dévalorisante. On ne peut plus guère voir là un effet du pétainisme. Il semble plutôt qu'il y ait rejet d'un mouvement dont l'existence semble contrevenir à la représentation légitime de la littérature française. Un critique littéraire le soulignait dès 1946:

Toutes les données du problème (le rejet du régionalisme) ne se raccordent pas à Vichy ${ }^{15}$. Il importe d'ailleurs de voir que comme source d'inspiration provinciale, le régionalisme n'a jamais produit que des œuvres de second ordre. Il relève d'un romantisme très subalterne et se situe en dehors de la grande tradition universaliste de l'esprit français. Au fond, il y a peu de genres moins français que le roman régionaliste ou la poésie de terroir. ${ }^{16}$

Et le même critique, en réponse aux protestations des régionalistes, réaffirme, dans un article ultérieur cette condamnation sans appel:

En ce qui concerne l'universalisme français, on ne contestera pas, même en tenant compte de Mistral qui n'écrivit pas Mireille en français, qu'il constitue la valeur dominante de notre littérature et que, dans le rayonnement de celle-ci à travers le

15 Vichy est la dénomination métonymique du gouvernement pétainiste, installe dans la ville de Vichy.

16 André Billy, Est-ce la fin du régionalisme? *, Le figaro littêraire, samedi 29 juin 1946. 
monde, le régionalisme n'a guère compté. Il y a des régionalismes dans tous les pays du monde, et souvent bien antérieurs aux nôtres. Il n'y a qu'un classicisme, et il est français ou d'inspiration française. Une vertu de l'existentialisme est d'avoir l'audience du monde intellectuel entier. ${ }^{17}$

Ce rejet du régionalisme, qui dérogerait à la vocation universaliste de la littérature française, est on ne peut plus manifeste dans les Histoires de la littérature française publiées depuis la dernière guerre mondiale. Le mouvement régionaliste forme l'un des chapitres absents, ou escamotés, de ces Histoires ${ }^{18}$. Et lorsque le terme est évoqué à l'occasion de la présentation d'un écrivain, c'est pour être aussitôt rejeté comme injustement péjoratif. Si l'auteur en question, précise l'auteur de la notice biographique, a mis en scène la vie d'individus dans un lieu précis, il a su en fait dépasser ce cadre étroit pour analyser les rapports (universels et a-sociaux) entre l'Homme (universel) et la Nature (a-sociale et an-historique). L'entrée dans l'histoire officielle de la littérature française est au prix de cette abstraction.

La rusticité des paysans de Giono semble à l'opposé de cette intelligence élégante, de ce raffinement de culture et de civilisation qui miroite dans l'écriture de Giraudoux. Pourtant, n'estce pas, par d'autres moyens, un même contact existentiel avec le monde qu'il $s$ 'agit de nouer? ${ }^{19}$

Colette, Ramuz, Giono: tant par leur structure que par leur éthique les romans de ces écrivains dépassent le régionalisme. 20

Un autre phénomène peut expliquer la perte, par le régionalisme, de la reconnaissance temporairement conquise: le développement de ce que l'on appelle les sciences humaines. La fiction réaliste n'a pas perdu les faveurs du public, mais les publications de sciences humaines, de plus en plus nombreuses et diversifiées, ont disputé la place tenue par le "roman docu-

17 André Billy, «Encore un mot sur le régionalisme, Le figaro littéraire, samedi 20 juillet 1946.

18 Le contraste est remarquable avec les Histoires de la littérature germanique publiées dans les dernières décennies, qui consacrent de nombreuses pages à l'équivalent allemand du régionalisme, la Heimatkunstbeuegung.

19 E. Carassus, dans Histoire littéraire de la France, tome 4 (1913-1976), Paris, Editions sociales, 1982, p. 482.

20 Ibid., p. 478. 
64

mentaire * auprès des lecteurs cultivés. Or depuis quelques décennies, les anciens domaines de prédilection du régionalisme ont fait l'objet d'expéditions et de conquêtes scientifiques. La sociologie rurale, l'ethnologie de la France, une certaine *histoire des mentalités" exposent les thèmes habituels du roman régionaliste. Les ouvrages qui en sont résultés, surtout lorsque leur style tendait à une certaine narrativité, ont pu atteindre des tirages importants. Depuis le début des années 1980 , nombre de librairies et de bibliothèques publiques provinciales présentent un rayon régionaliste: mais on y trouve surtout des publications (ou republications) à caractère historique et ethnographique.

L'histoire du régionalisme, dans ses avatars, montre en fait la force avec laquelle s'impose la norme esthétique française. Le principe de l'universalisme constitue la forme légitimée du sociocentrisme et de l'ethnocentrisme. La prééminence accordée à la culture d'une classe, d'une civilisation, qui la pose en mesure absolue a pour conséquence le rejet du particularisme ou sa relégation dans des positions inférieures. Par conséquent, la mise en cause de la hiérarchie culturelle se trouve tout naturellement associée à l'apologie du particularisme. Enfermée dans cette position dévalorisée, elle ne peut avoir de reconnaissance que dans des contextes spécifiques et temporaires, quand une crise globale de la société introduisant provisoirement le doute sur l'efficacité de l'ordre établi lui fournit un argumentaire idéologique $^{21}$.

21 On peut également observer que les littératures d'expression française - non hexagonales * (littératures du Québec, cu Maghreb, d'Afrique noire, des Antilles, de Belgique, de Suisse romande etc.) sont par périodes l'objet d'engouement et remportent des prix littéraires en France, mais elles ne sont pas véritablement intégrées dans l'histoire de la littérature française ni dans les programmes d'enseignement littéraire. 